\title{
Effect of high-voltage electrostatic field on cryopreservation of human epidermal melanocytes
}

\author{
Xu Cai ${ }^{1}$, Pengcheng $\mathrm{Li}^{1,2}$, Benke Chen ${ }^{1}$, Qian $\mathrm{Li}^{1}$, Jintian Tang ${ }^{1 *}$, and Xianwei Zou ${ }^{1{ }^{* a}}$ \\ ${ }^{1}$ Key Laboratory of Particle \& Radiation Imaging, Ministry of Education, Department of Engineering Physics, Tsinghua University, \\ Beijing, China \\ ${ }^{2}$ College of Food Science and Engineering, Jilin Agricultural University, Changchun, China
}

\begin{abstract}
The objective of this research was to evaluate the influence of high-voltage electrostatic field (HVEF) on the freezing of human epidermal melanocytes (HEM) with respect to the degree of cell deformation, survival and proliferation rate after cell resuscitation. As a result, the degree of supercooling is increased by enhancing the strength of the static electric field in the range of $15 \mathrm{kV} / \mathrm{m}$, and the maximum supercooling degree is $7.83 \pm 0.05^{\circ} \mathrm{C}$ at $15 \mathrm{kV} / \mathrm{m}$. By contrast, the morphology of the electric field assisted freezing cell after resuscitation was significantly improved, and the best electric field strength for cryopreservation is $15 \mathrm{kV} / \mathrm{m}$. The survival rate of human epidermal melanocytes recovered was $88.03 \%$, which was higher than that of the control group. The proliferation rate at 24,48 and 72 hours are $17 \%, 28 \%$ and $25 \%$, respectively, which are higher than that of the control cells. These findings demonstrate that the freezing HVEF can protect the cell physiological activity, and reduce the freezing damage. Therefore, the optimal HVEF cryopreservation technology be of great significance for the research of tissue engineering in repairing wounds, infections, and promote the development of food, medicine and other fields.
\end{abstract}

\section{Introduction}

The living cells are often used as research objects in modern medical treatments. Consequently, it is important for preserving and maintaining the activity and function of various types of cells $[1,2]$. Cooling cells at a sufficiently low temperature can reduce their metabolism and preserve them for a long time. To improve the effect of cell cryopreservation, cytoprotective agents, such as glycerol, ethylene glycol, dimethyl sulfoxide, propylene glycol, were added. However, the toxicity of cytoprotective agents could affect the cell activity [3, 4]. Meanwhile, osmotic pressures and ice crystals can cause solute damage and mechanical damage to cells or tissues in the process of freezing [5]. Some physical techniques are used to help cells freeze to better preserve them. Frank reduced the precipitation of salt ions in the freezing process by highpressure technology, thus avoiding the damage of ion osmotic pressure to cells [6]. Ackerton acted on sucrose solution by ultrasonic pulse, which reduced the crystal size of ice crystals [7]. Jackson has proposed and verified that microwave-assisted cryoprotectants can inhibit the formation of ice crystals in the cooling process [8].

High External electrostatic field (HVEF) has great potential in the new cryopreservation technology. The studies have shown that the structure of water clusters can be changed and the formation of ice crystals can be reduced under the electrostatic field $[9,10]$. Xanthakis applied electrostatic field to freeze pork, and observed that the size of ice crystals in pork decreased with the increase of voltage[11]. Hsieh compared the effects of electrostatic field assisted thawing and ordinary thawing on chicken thigh meat [12]. HVEF can both accelerate the freezing and thawing speed of meat, and reduce the rate of juice loss, the damage of meat tissue and muscle fibers in the thawing process [11]. Tomoki used electrostatic field to preserve red blood cells, which can improve the survival time of red blood cells and maintain the physiological activity of red blood cells [13]. Therefore, the HVEF has significant effects on the preservation of biological tissues. However, few studies have been done on solution crystallization and cell preservation by HVEF. Therefore, we designed the study to investigate the influence of static electric field on the freezing of normal human epidermal melanocytes (HEM) cell.

\section{Methods}

\subsection{Apparatus and Chemicals}

HVEF consists of a pair of parallel plate electrodes, a high-voltage power and a cooling system. Fluorescence fiber optic thermometer (Xi'an photoelectric technology Co. LTD, China), Inverted microscope (Olympus, Japan), Varioskan flash microplate reader (Thermo fisher, USA),

\footnotetext{
${ }^{a}$ Correspondence author: Jintian Tang, E-mail, tangjt@mail.tsinghua.edu.cn
} 
HEM (National center cell bank introduction, Chian), DMSO, FBS, DMEM, MTT, Giemsa stain (Sigma, USA)

\subsection{Determination of supercooling degree of cell freezing medium under different conditions}

The output voltage of transmission power is adjusted to make the field intensity $10,15,20,25,30,40$ and 60 $\mathrm{kV} / \mathrm{m}$, and freeze at $-8,-10$, and $-12{ }^{\circ} \mathrm{C}$, respectively. The optical fiber is arranged to insert the into the conical bottle and fix it in the center of cell freezing medium (DMEM:FBS:DMSO = 6:3:1). The temperature of sample center is recorded in real time by the optical fiber thermometer. When the central temperature of sample rises from the supercooled state temperature to the freezing point temperature of cryoprotectant solution, it is considered that the freezing is completed, and the absolute value of this temperature is the super cooled degree of saline solution. The time spent in freezing solution from $4{ }^{\circ} \mathrm{C}$ to this point is the freezing time of solution.

\subsection{Morphological observation of HEM frozen with HVEF}

The cell were assisted by HVEF to cryopreserve (10, 15 and $20 \mathrm{kV} / \mathrm{m}$. After 30 minutes of cooling at $4{ }^{\circ} \mathrm{C}$, the cells were transferred to $-10{ }^{\circ} \mathrm{C}$ for 30 minutes, then transferred to $-20{ }^{\circ} \mathrm{C}$ for one hour. After being stored in liquid nitrogen at $-80{ }^{\circ} \mathrm{C}$ for three days, the cells were taken out for resuscitation. The cells of were melted in water bath at $37^{\circ} \mathrm{C}$, washed with PBS, and stained with Giemsa stain for 5-10 minutes, then rinsed three times with water. After drying, the cells were observed under inverted microscope.

\subsection{Measurement of cell viability before and after cryopreservation with HVEF}

The cells were inoculated into 96-well plates with a density of $1.5 \times 10^{4}$ cells $/ \mathrm{mL}$ and $200 \mu \mathrm{L}$ per hole. The cells were cultured in cell incubator. After 24 hours, the $10 \mu \mathrm{L}$ MTT solution was added to each hole, and then cultured in incubator. After 4 hours, culture solution was discarded, and $150 \mu \mathrm{L}$ DMSO solution was added to each hole. The OD value was measured at $490 \mathrm{~nm}$ by varioskan flash microplate reader.

$$
\text { Cell viability } \%=\frac{A_{\text {pre-freezing }}}{A_{\text {cryopreserved }}} \times 100 \%
$$

\subsection{Statistical analysis of data}

The data were analyzed by using SPSS 21.0, and were expressed as $\bar{x} \pm s$.

\section{Results}

\subsection{Effect of the HVEF on the nucleation temperature and on the supercooling degree}

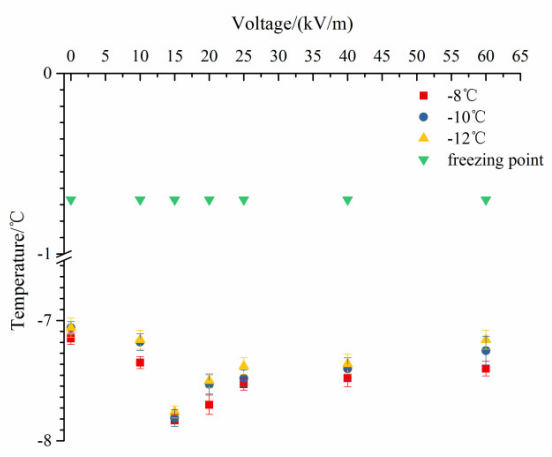

Figure. 1. Averages of nucleation temperature $(\mathrm{Tn})(\bullet, \mathbf{\square}$ and $\boldsymbol{\Delta})$ and freezing point $(\boldsymbol{\nabla})$ with their standard deviations of pork tenderloin samples under different static electric fields.

The effect of HVEF on the supercooling degree of cryoprotectant solution at different freezing temperatures is shown in Figure. 1. Compared with the control group, the nucleation temperature of the solution in the HVEF groups reduced to a certain extent, but the freezing point of the solution was not affected by the applied HVEF. It is exclusively governed by the decreased in the nucleation temperature. Under the HVEF of $15 \mathrm{kV} / \mathrm{m}$, the nucleation temperature decreased by $0.67{ }^{\circ} \mathrm{C}(\mathrm{p}<$ $0.05)$. Our experimental findings are in agreement with previous work by Orlowska [14]. The reason for the increase of supercooling may be that the Gibbs free energy of water molecules was changed by applied HVEF. According to the calculation method proposed by Marand [15], the free energy expression of the crystal nucleus in the presence of electric field is as follows:

$$
\Delta G_{E}=4 \pi r^{2} \gamma-\frac{4}{3} \pi r^{3}\left(\Delta G_{V}+P E\right)
$$

The factors of r, $\gamma, \Delta G_{E}, \Delta G_{V}, \mathrm{E}, \mathrm{P}$ are radius of crystal nucleus sphere, surface free energy of crystal fluid interface, crystal nucleus free energy, free energy change per unit volume, external electrostatic field, and dipole moment, respectively. It can be seen from formula (2) that the application of electrostatic field can decreases the free energy when the solution is frozen. The nucleation temperature of solution increased with the increase of field strength when the strength of the electric field was greater than $15 \mathrm{kV} / \mathrm{m}$. The reason for the decrease of supercooling does not meet Gibbs free energy rules by applied stronger HVEF. In addition, the nucleation temperature is similar under different freezing temperatures.

\subsection{Effect of the HVEF on freezing time of frozen storage fluid}

The effect of HVEF on freezing time of cryoprotectant solution was showed in Table. 1. The result shows that 
the freezing time of solution is significantly increased by applying different strong HVEF, in which the freezing time of solution is maximum at $15 \mathrm{kV} / \mathrm{m}(\mathrm{p}<0.05)$. When the electric field intensity is greater than $15 \mathrm{kV} / \mathrm{m}$ $(p<0.05)$, the freezing time of solution gradually. When

Table. 1 Freezing time of cryoprotectant solution at cooling temperatures of $-8,-10$ and $-12{ }^{\circ} \mathrm{C}$ under different static electric fields.

\begin{tabular}{llll}
\hline Groups $(\mathrm{kV} / \mathrm{m})$ & $-8^{\circ} \mathrm{C}$ & $-10^{\circ} \mathrm{C}$ & $-12^{\circ} \mathrm{C}$ \\
\hline 0 & $33.71 \pm 3.38$ & $25.84 \pm 1.47$ & $21.59 \pm 1.79$ \\
10 & $40.99 \pm 2.20^{*}$ & $34.53 \pm 2.22^{*}$ & $24.85 \pm 1.55^{*}$ \\
15 & $52.99 \pm 2.22^{*}$ & $43.13 \pm 2.26^{*}$ & $26.63 \pm 0.75^{*}$ \\
20 & $45.07 \pm 3.35^{*}$ & $39.49 \pm 2.19^{*}$ & $25.90 \pm 0.29^{*}$ \\
25 & $43.55 \pm 3.30^{*}$ & $38.15 \pm 2.43^{*}$ & $24.95 \pm 1.54^{*}$ \\
40 & $42.86 \pm 2.31^{*}$ & $35.95 \pm 1.31^{*}$ & $24.79 \pm 0.58^{*}$ \\
60 & $41.05 \pm 1.21^{*}$ & $34.00 \pm 1.49^{*}$ & $25.17 \pm 1.44^{*}$ \\
\hline
\end{tabular}

the electric field intensity is greater than $15 \mathrm{kV} / \mathrm{m}(\mathrm{p}<$ 0.05 ), the freezing time of solution gradually decreases with the increase of electric field intensity. The results showed that the different strong HVEF had different effects on freezing time of cryoprotectants. In addition, the freezing time of the solution was prolonged by $57.19 \%, 62.91 \%$ and $12.88 \%$ at $-8,-10$ and $-12{ }^{\circ} \mathrm{C}$ at 15 $\mathrm{kV} / \mathrm{m}$, respectively. And with the decrease of the freezing temperature, the freezing time of the solution decreased gradually. The final result of slow freezing process is the shrink of cells surrounded by ice crystals. With the intervention of osmotic cryoprotectants, the cells are usually in glass solid or partially crystallized glassy media. The more cryoprotectants can fully enter cells during cryopreservation, which can inhibit the formation of intracellular ice crystals, and improve the survival rate of cells in cryopreservation.

\subsection{Effect of the HVEF on the morphology of HEM}

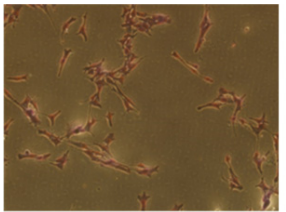

Control (20x)

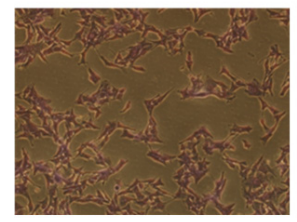

$15 \mathrm{kV} / \mathrm{m}(20 \mathrm{x})$

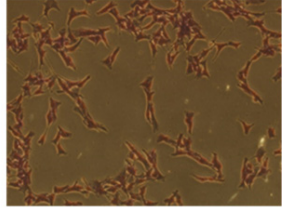

$10 \mathrm{kV} / \mathrm{m}(20 x)$

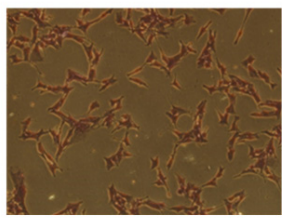

$20 \mathrm{kV} / \mathrm{m}(20 \mathrm{x})$
Figure. 2 Giemsa staining of HEM.

In micrographs images of HEM under different freezing conditions are presented (Figure. 2). The control group were damaged and changed in morphology, such as slender, more branches and poorer cell adherence. Compared with the control group, the experimental group showed obvious improvement in cell morphology.
And cells treated with $15 \mathrm{kV} / \mathrm{m}$ showed the good growth, clear outline, normal morphology and firm adherence, and the increase cell density. Meanwhile, the dendritic structure of cells was obtuse, which indicated that the cells with less damaged and grow well. Therefore, it can be inferred that electro-freezing can improve the cryopreservation of cell.

\subsection{Effect of the HVEF on survival rate of HEM after resuscitation}

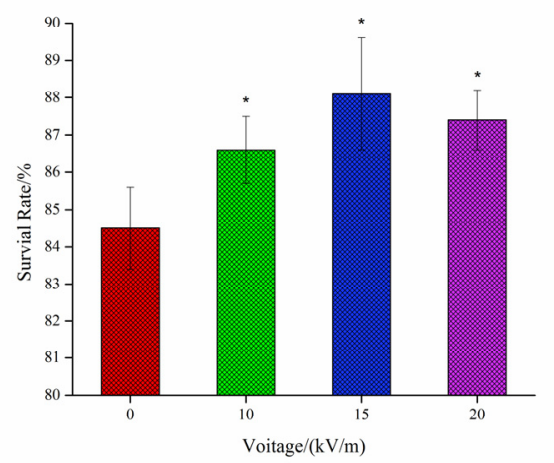

Figure. 3 Survival rate of HEM treated with different magnitude static electric fields.

When treated with $10 \mathrm{kV} / \mathrm{m}, 15 \mathrm{kV} / \mathrm{m}$ and $20 \mathrm{kV} / \mathrm{m}$, the cell survival rate was $86.66 \%, 88.03 \%$ and $87.41 \%$, respectively. There was significant difference between the control group and the experimental group $(\mathrm{p}<0.05)$, which showed that the HEM survival rate cryopreservated with HVEF was improved (Figure. 3). Because the higher supercooling degree of solution with the longer freezing time, the survival rate of cells after resuscitation is increased. Therefore, the cryopreservation of cell can obtain higher survival rate by applying electric field.

\subsection{Effect of the HVEF on the growth curve of HEM after resuscitation}

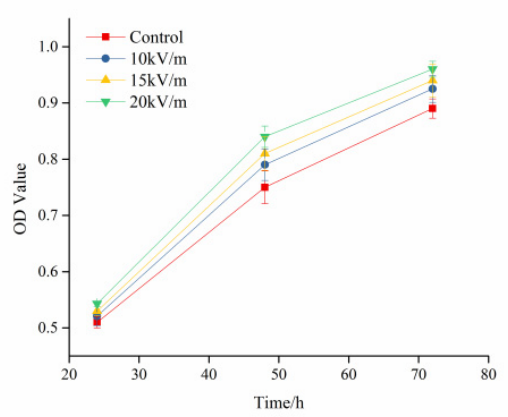

Figure. 4 Growth curve of HEM treated with different static electric fields

The growth trend of the four groups was showed in Figure. 4. Compared with the control group, the growth rate of the experimental group is faster. There were significant differences under $15,20 \mathrm{kV} / \mathrm{m}$ after 72 hours 
of resuscitation $(\mathrm{p}<0.05)$. The statistical results show that the cryopreservate of HEM with HVEF could promote the growth state of cells after recovery with better activity and faster proliferate. Among them, the effect is best under $15 \mathrm{kV} / \mathrm{m}$.

\section{Conclusions}

The study of HVEF shows that HVEF could significantly increase the supercooling degree and freezing time of solution, and protect cell morphologyt. After resuscitation, cells with clear boundaries, lower deformations, stronger adherence which is related to the biological function of cells. This paper discussed the effect of electric field freezing on cell survival rate after recovery. Under the condition of electric field freezing, cell survival rate is significantly improved. It is speculated that ice crystal has less damage to cells and reduces cell damage in the process of freezing. It was found that HVEF could increase the proliferation rate of cells by plotting the growth curve of cells after freezing with HVEF, which again showed that cells were better protected in the process of freezing and improve the tolerance of normal cells after resuscitation. This experiment focuses on the changes of cell survival rate, morphology and growth curve after resuscitation, while it needs to be further studied the effects of electric field on cell function. These findings demonstrate clearly the advantage of freezing under HVEF that can protect the physiological activity of the cells, reduce the freezing damage. HVEF is a promising and innovative freezing process, and could promote the development of food, medicine and other fields.

\section{Acknowledgements}

This work was supported by the National Natural Science Foundation of China (21402104), and the Project funded by China Postdoctoral Science Foundation (2014T70068).

\section{References}

1. J. Karlsson, K. Toner, Principles of tissue engineering, 2:293-307, (2000).

2. R. Langer, J. Vacanti, Sci, 260: 920-926, (1993).

3. B. Wowk, E. Leitl, C.M. Rasch, N. Mesbah-Karimi, S.B. Harris, G.M. Fahy, Cryobiology, 40(3):228-236, (2000).

4. H.S. Ren, Y. Wei, T.C. Hua, J. Zhang, Cryobiology, 31(1):47-56, (1994).

5. P. Mazur, S.P. Leibo, E.H. Chu, Exp Cell Res, 71(2):345-355, (1972).

6. M.R. Frank, C.E. Rungeb, H.P. Scott, S.J. Maglio, J. Olson, V. B. Prakapenka, G.Y. Shen. Phy Earth and Planet In, 155(1-2):152-162, 2006.

7. J.P. Acker, A. Larese, H. Yang, A. Petrenko, L.E. McGann, Cryobiology, 38(4): 363-371, (1999).

8. T.H. Jackson, A. Ungan, J.K. Critser, D. Gao,
Cryobiology, 34(4):363-372, (1997).

9. D. Jung, J. Yang, M. Jhon, Chem Phys, 244, 331337, (1999).

10. S.V. Shevkunov, A. Vegiri, J Mol Struc Theochem, 593(1):19-32, (2002).

11. E. Xanthakis, M. Havet, S.C. hevallier, J. Abadie, A. Le-Bail, Innov Food Sci Emerg, 20, 115-120, (2013)

12. C.W. Hsieh, C.H. Lai, W.J. Ho, S.C. Huang, W.C. Ko, J Food Sci, 75(4), 193-197, (2010).

13. T. Nishiyama, D. Hayashi, J Anesth, 21(1):42-46, (2007)

14. Sun Y, Yan L, Jin G, et al. Chinese journal of medical physics, 22(1), 391-392, (2005).

15. H. Marand, R. Stein, G. Stack, J Polym Sci Pol Phys, 26, 1361-1383, (1988). 